\title{
La jornada continua como un recurso para la maternidad intensiva. Una mirada desde la Comunidad Valenciana
}

\author{
The Compacted School Schedule as A Resource for Intensive Mothering. \\ A Look From the Valencian Region
}

Sandra Obiol-Francés ${ }^{1}$

\begin{abstract}
Resumen
La «ideología de la maternidad intensiva» (Hays, 1998) según la cual la prioridad de la madre es dar respuesta a las necesidades de los hijos e hijas mediante la inversión de grandes cantidades de recursos económicos, temporales y emocionales, proporciona en este artículo una perspectiva clarificadora para comprender mejor el apoyo de un gran número de familias a la jornada continua en las escuelas. El artículo representa una primera aproximación a la relación entre maternidad intensiva y jornada escolar, a partir de la reflexión teórica que suscita la bibliografía existente sobre la construcción social de la maternidad, los análisis realizados sobre la jornada escolar en el ámbito español y materiales generados por los centros educativos. Los resultados obtenidos, aunque tentativos, apuntan a que la jornada continua está jugando un papel importante en la distinción de las familias más acomodadas, con mayores recursos para poder maximizar el tiempo que deja la compactación horaria. Al mismo tiempo supone un fortalecimiento de la responsabilidad individual, en este caso de las madres especialmente, que conlleva la individualización característica de la modernidad reflexiva y que puede generar un incremento de la desigualdad.
\end{abstract}

\section{Palabras clave}

Maternidad intensiva, jornada escolar, género, individualización, tiempo.

\section{Abstract}

The «ideology of intensive mothering» (Hays, 1998), according to which the mother's priority is to respond to the needs of her children by investing large amounts of economic, temporal and emotional resources, provides in this article a clarifying perspective to better understand the support of a large number of families for the continuous school day. The article represents a first approach to the relationship between intensive mothering and the school day, based on theoretical reflection on the existing bibliography on the social construction of motherhood, the analyses carried out on the school day in Spain and materials generated by educational centers. The results obtained, although tentative, point to the fact that the continuous school day is playing an important role in distinguishing the more affluent families, with greater resources to maximize the time left by the compact timetable. At the same time, it implies a strengthening of individual responsibility, in this case of mothers in particular, which entails the individualization characteristic of reflexive modernity and which may generate an increase in inequality.

\section{Keywords}

Intensive mothering, school day, gender, individualization, time.

\section{Cómo citar/Citation}

Obiol-Francés, Sandra (2021). La jornada continua como un recurso para la maternidad intensiva. Una mirada desde la Comunidad Valenciana. Revista de Sociología de la Educación-RASE, 14 (3), 291-306. http://dx.doi.org/10.7203/RASE.14.3.21545. 


\section{Introducción}

En la década de los 90 surgió en algunas Comunidades Autónomas la demanda de la compactación horaria del tiempo lectivo durante el horario de la mañana, eliminando la pausa de mediodía y concentrando las 5 horas lectivas durante la mañana, de 9h. a 14h. En un primer momento esta demanda se focalizó en la educación secundaria, donde hoy ya es el modelo mayoritario, para más tarde ir ampliándose a los centros de educación primaria e infantil. Según los datos recogidos en estudios anteriores (Gabaldón-Estevan y Obiol-Francés, 2017) la extensión de este modelo se inicia en las Islas Canarias (1992) y Galicia (1993) para progresivamente alcanzar a casi la totalidad de las autonomías excepto Catalunya, País Vasco y Cantabria. Sin embargo, también en estos territorios se ha puesto sobre la mesa, recurrentemente, el debate acerca la conveniencia del cambio de organización horaria, en especial a partir de los cambios organizativos la Covid-19 ha obligado a introducir en los centros educativos.

A pesar de su amplia expansión, el modelo de jornada escolar carece de evidencias científicas que la avalen, más bien al contrario. Los pocos análisis que existen desaconsejan su aplicación por tratarse de una organización horaria contraria a los ritmos de aprendizaje de los niños y niñas además de una fuente de desigualdad (Feito, 2007; Sintes, 2012; Fernández Enguita, 2001; Morán de Castro y Cruz, 2011; Morán de Castro, 2012; Gabaldón-Estevan y Obiol-Francés, 2017). Pero todas estas evidencias en contra no han impedido que la jornada escolar continua se haya instalado de manera mayoritaria en España estructurando el tiempo de miles de familias.

La Comunidad Valenciana se incorpora recientemente a este proceso de cambio. En junio de 2016 se publica el decreto ${ }^{2}$ que regula la posibilidad de los centros educativos de solicitar el cambio de jornada escolar y, aunque la normativa no indica cómo ha de ser esta jornada, la totalidad de las solicitudes suponen la concentración de las horas lectivas durante el periodo matinal. Había existido un precedente en forma de proyecto piloto bajo el gobierno del Partido Popular para un total de nueve centros, que no se extendió al conjunto de los centros valencianos a raíz de los resultados negativos de la evaluación interna realizada por la misma Conselleria d’Educació (Gabaldón-Estevan y Obiol-Francés, 2017). Sin embargo, con el cambio de gobierno de 2015 —en el que accedió al poder una coalición constituida por PSOE y Compromís - se volvió a retomar este cambio y se elaboró una normativa en la que se establecían las pautas para que durante el curso 2017-2018 los centros educativos valencianos pudieran decidir sobre el cambio en la estructuración de su jornada escolar. Desde ese momento el proceso de expansión ha sido constante, hasta el punto que según los datos disponibles en la página web ${ }^{3}$ de la Conselleria d'Educació, en septiembre de 2019, un total de 649 centros de educación primaria y/o infantil estaban autorizados para compactar su jornada, de los cuales la inmensa mayoría eran de titularidad pública, un 96,5\% (626). Estas cifras muestran que del total de centros públicos de educación primaria en la Comunidad Valenciana un $62,1 \%$ tienen jornada continua y un $6,23 \%$ entre los centros de titularidad privada ${ }^{4}$.

Ante estas cifras la primera pregunta que surge es: ¿por qué? No deja de ser desconcertante que a pesar de que todas las evidencias científicas y la experiencia internacional la desaconsejen, la jornada continua genere tantas adhesiones. Los estudios existentes exponen un debate acerca de la jornada escolar muy parecido allá donde se ha producido: con claros tintes corporativos, en el que los docentes persiguen más

\footnotetext{
Orden 25/2016, de 13 de junio de la Conselleria de Educación, Investigación, Cultura y Deporte, DOCV núm. 7806, 15.06.2016.

Listado accesible en: https://ceice.gva.es/documents/162640733/168106814/Centros+PEOJE+-+Setembre+2019.pdf/c7d75e35-841a-40e2-a7a7-e57ec03afaa9

4 Este peso debería también ser analizado a nivel municipal, pues nos topamos con poblaciones donde la jornada continua es minoritaria y otros donde afecta a toda su oferta pública.
} 
objetivos laborales que no pedagógicos, muy visceral y con una Administración que ha evitado posicionarse para sortear los costes políticos que puede comportar entrar en un juego de intereses tan complejo (Fernández Enguita, 2001; Feito, 2007; Morán de Castro, 2012). Y en este debate a las familias se les ha otorgado, generalmente, un papel secundario, a expensas de las decisiones tomadas por los docentes, aunque es complicado negar el hecho de que la mayoría de las familias han entendido que la jornada continua les beneficia, a ellas y a sus hijos. El caso valenciano lo hace evidente: sin el apoyo mayoritario de las familias ninguno de los centros hubiera podido modificar su organización horaria. No en vano los fundamentos reguladores del cambio de jornada en la Comunidad Valenciana reclamaban, como primer trámite, la elaboración por parte de cada centro educativo de un proyecto — Plan Específico de Organización de la Jornada Escolar - en el que se plantearan los principales motivos que fundamentaban el cambio junto a la nueva organización horaria y sus efectos sobre el alumnado, el profesorado y las familias. El plan debía de contar con la aprobación mayoritaria de todos y cada uno de los agentes sociales que conforman la comunidad educativa: el consejo escolar municipal, el claustro, el consejo escolar de centro y un mínimo del $55 \%$ de votos favorables de la totalidad del censo de madres y padres. Este proceso de modificación horaria por fases ha generado un intenso debate en el que el grado de cohesión de las diferentes comunidades educativas, el nivel de implicación de los gobiernos locales, la capacidad de movilización de las direcciones de los centros y de sus claustros en esta lucha dibujan escenarios diferentes, con diferentes niveles de conflicto ${ }^{5}$. Pero la última palabra en el proceso establecido por la Generalitat Valenciana para el cambio de la jornada escolar corresponde a las familias. Sólo si la propuesta de modificación de la jornada es aceptada mayoritariamente por las familias censadas puede llevarse a cabo.

Comprender mejor las razones por las que las familias han optado de manera mayoritaria en los centros públicos valencianos por aceptar, incluso promover, el cambio horario es el propósito de este artículo. He considerado útil para ello ampliar la mirada y entender a la escuela no en sí misma, sino como un elemento más de un complejo conjunto de recursos que las familias tienen a su disposición para cumplir con la que, con el paso del tiempo, se ha convertido en su principal función: la crianza y socialización de los hijos/as. Entender la escuela como una pieza más del complicado rompecabezas que supone en la sociedad occidental actual considerarse una buena madre, un buen padre, nos aporta una perspectiva diferente y clarificadora ante la jornada continua. Nos permite, a mi juicio, calmar el desconcierto que genera la defensa de un cambio en pro de unos beneficios que, según todos los indicadores existentes, no se consiguen. Ser unos buenos padres, especialmente ser una buena madre, implica una serie de convenciones sociales cada vez más absorbentes y exigentes, que requieren de un esfuerzo a veces extenuante. Compartimentar el tiempo que los niños/as y jóvenes pasan en el centro educativo y despejar así las tardes para poder cumplir con estas exigencias es un argumento que se ha de considerar para poder entender mejor la expansión de la jornada escolar continua y, también, el alcance de sus implicaciones, sobre todo en términos estructurales.

El presente artículo es el resultado de una primera aproximación a la extensión de la jornada continua en el Estado español, más en concreto en la Comunidad Valenciana. A partir de la bibliografía existente sobre la construcción social de la maternidad —y sus implicaciones en la estructura social— junto a los análisis realizados sobre la jornada escolar en el ámbito español y materiales generados por los agentes so-

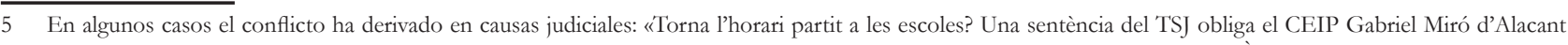
a revertir la jornada continuada i obri la porta perquè més de 300 centres educatius seguisquen el mateix camí». Laura López. À punt (02.05.2021). Accesible en: https://www.apuntmedia.es/noticies/societat/torna-l-horari-partit-escoles_1_1415694.html. 
ciales implicados, en especial los centros docentes, trato de construir un marco desde el cual comprender mejor las razones por las que se defiende la conveniencia de la jornada continua desde la consideración cultural acerca de lo que representa ser una buena madre y, por extensión, el buen cuidado de los hijos/as.

El texto se divide en tres grandes apartados. En primer lugar, defino de qué hablamos cuando lo hacemos de la ideología de la maternidad intensiva. En segundo lugar, me centro en el proceso de cambio de jornada en la Comunidad Valenciana y los principales argumentos argüidos, centrándome en aquellos que defendían ampliar el tiempo de los niños/as fuera de la escuela. Para terminar, apunto algunas reflexiones acerca la relación entre la ideología de la maternidad intensiva y la jornada escolar continua.

\section{La maternidad intensiva en el fondo del debate sobre el tiempo escolar}

La discusión acerca de la conveniencia del cambio en la jornada escolar ha sido encabezada por los partidarios de la reforma a partir de un conjunto de argumentos, muy homogéneos entre sí que han conseguido extenderse de manera apabullante y conseguir una mayoritaria y sólida adhesión a pesar de no contar con una fuente clara que los recopile de manera sistemática. Estos argumentos hacen referencia, fundamentalmente, a la mejora del rendimiento académico, a la disminución de la fatiga y del cansancio del alumnado, a la posibilidad de favorecer la organización de la vida fuera de la escuela (pudiendo dedicar más tiempo a la familia y a las actividades extraescolares), a la capacidad de organización del profesorado (mejorando la atención a las familias y ampliando su tiempo de formación), y al elevado grado de satisfacción que ha conseguido este tipo de jornada allá donde se ha instalado (Feito, 2007: 76; Sintes, 2012). En consecuencia, una parte de los argumentos a favor del cambio de jornada escolar se ha sustentado en la necesidad de despejar las tardes de la presencialidad del tiempo lectivo. Con la tarde sin las obligaciones de la educación formal, los niños y niñas podrían ampliar el tiempo que pasan en familia a la vez que podrían asistir a un mayor número de actividades extraescolares, o bien que todas estas actividades pudieran realizarse de manera más pausada. Así sería más sencillo poder invertir en actividades consideradas beneficiosas para el desarrollo emocional y educativo de los niños/as, una preocupación central para los progenitores, especialmente las madres, si se tiene en cuenta la llamada «ideología de la maternidad intensiva».

\subsection{La tarea de convertirse en una buena madre}

A mediados de la década de los 90, y para dar nombre al conjunto de normas culturales acerca del cuidado infantil óptimo en la sociedad norteamericana, pudiéndose extender a la sociedad occidental en su conjunto, Hays (1998) acuña el término de «ideología de la maternidad intensiva». Construida y avalada por el discurso mayoritario procedente de expertos en cuidado infantil esta ideología identifica a la madre como cuidadora prioritaria, la única capaz de crear un ambiente lo suficientemente cálido y protector para dar respuesta a las necesidades de los hijos/ as, invirtiendo para ello grandes cantidades de recursos económicos, temporales y emocionales. Según estas ideas, el propósito principal de la madre es la protección de los niños/as ante los peligros de la sociedad, presentes y futuros, proveyéndoles de todos los recursos que intuyen necesarios para que se conviertan en adultos autónomos, responsables, adultos de éxito, en definitiva, con toda la incertidumbre que este objetivo supone, tanto en la definición del éxito como en la de los recursos necesarios. Y este — difuso- propósito ha de marcar las decisiones que tome la madre, en relación a sus hijos e hijas y, claro está, en relación a su propia vida. 
A partir del siglo XviII se va consolidando la idea que el destino natural de las mujeres sea dar respuesta a las necesidades de los hijos en el marco del hogar, siempre con la guía de los expertos (científicos y médicos), defendiendo nuevas recetas de crianza y arrebatando el control de este cuidado de las manos de las que habían sido sus responsables, las mujeres (Bolufer, 1995, 2003, 2010; Rich, 1996). Esta concepción de la maternidad como destino total de la mujer se consolidó con la separación de las esferas pública y privada que supuso la industrialización, y que impuso como ideal a conseguir la dedicación en exclusiva de la mujer en el ámbito privado donde la maternidad y el cuidado eran sus actividades primordiales (Aguado, 1998). A pesar de que el movimiento feminista en los 70 cuestionó claramente la maternidad como destino para las mujeres y hoy en día la maternidad es considerada una opción para la mayoría de las mujeres en la sociedad occidental, las actuales normas de crianza y cuidado requieren de una atención constante y completa de las madres convirtiéndolas en madres a tiempo completo (Badinter, 2011). Precisamente por tratarse la maternidad de una opción, sus requerimientos resultan más rígidos, con la asunción central de que la presencia de la madre es insustituible (Edgley, 2021).

En el caso español los medios de comunicación, con la contribución decisiva de las redes sociales, han diseminado estas recetas intensivas de crianza, en las que las madres - las buenas madres - han de atender prioritariamente las necesidades de sus hijos/as, respetando los ritmos que ellos mismos marquen. Es los que nos muestra el estudio realizado por Medina-Bravo et al., (2014) sobre las revistas de crianza y familia de mayor consumo como elementos de socialización en esta ideología de la maternidad intensiva. Junto a las publicaciones periódicas, las redes sociales son una fuente inagotable de recetas, a veces contrapuestas (del Olmo, 2013), pero que se sustentan en la individualización del cuidado y en la presencia del mercado como proveedor fundamental de los instrumentos para este cuidado. Son recetas congruentes con la aceleración y profundización del proceso de individualización entendida como la toma de conciencia de que nosotros mismos somos responsables de nuestra vida (Beck y Beck Gernsheim, 2003) y, en consecuencia, somos los principales responsables del bienestar y de las oportunidades vitales de nuestros hijos e hijas. Sin embargo, es preciso anotar que en los últimos años, sobre todo con la proliferación de las plataformas de contenidos de ficción en streaming así como con el mayor acceso de las mujeres a equipos creativos, están apareciendo nuevas miradas hacia la maternidad que se alejan de la visión tradicional y que, entre otros aspectos, muestran los diferentes intereses de las mujeres que han sido madres más allá de la crianza (Visa, 2019). También es remarcable la fuerza que ha tenido la blogosfera de madres para difundir nuevas miradas sobre la crianza y la maternidad (Visa y Crespo, 2012).

Y aunque las mujeres han jugado un papel trascendente en este proceso de individualización incluida en la llamada modernización reflexiva, ampliándose sus expectativas vitales más allá de la formación de una familia (Beck-Gernsheim, 2003) el hecho es que perdura la mayor responsabilidad de las mujeres en las relaciones familiares y de cuidado. Es innegable que, como señalan Barbeta y Cano (2007), se está produciendo un cambio en el papel que los hombres asumen como cuidadores, desde el modelo tradicional de paternidad, de una gran homogeneidad, hacia una concepción amplia, plural y multidimensional de la experiencia paternal de los hombres. Los hijos/as, según estos autores, ocupan cada vez más un lugar central en la construcción de la identidad masculina y se legitima su implicación directa en las actividades rutinarias de 
los hijos/as, también en la reinterpretación bajo el prisma de la crianza intensiva de elementos tradicionales de la paternidad (como el sustento económico) (Shirani et al., 2011). Sin embargo, son las mujeres las que se encargan en mayor medida de estas actividades de cuidado, especialmente aquellas que requieren mayor grado de implicación. Sin menospreciar la importancia del avance hacia una paternidad más presente en el cuidado cotidiano, así como la trascendencia en el debilitamiento de la masculinidad hegemónica que supone asumir prioritariamente por parte de los padres el cuidado de los hijos/as la posibilidad de tomar por parte de los hombres de permisos parentales (Romero-Balsas, Meil y Rogero-García, 2021) todavía hoy la crianza es un territorio protagonizado por mujeres. No sólo la cantidad de tiempo de las mujeres dedicado a las tareas de cuidado familiar supera a la de los hombres ${ }^{6}$, también su mayor opción a interrumpir su vínculo con el mercado laboral por cuestiones de cuidado ${ }^{7}$. Una centralidad que se ve acrecentada por el carácter familiarista de nuestro régimen de bienestar (Naldini, 2003). Y se ha visto incrementada por los efectos negativos que primero la Gran Recesión y sus políticas de austeridad, que han mermado los recursos destinados a políticas de igualdad al tiempo que han debilitado, más, la posición laboral de las mujeres (Gálvez, 2013; Gálvez y Rodríguez-Modroño, 2016). Una situación que todo indica que se ha acrecentado por la crisis generada por la Covid19 (Salido, 2021).

\subsection{La maternidad intensiva como fuente de desigualdad}

El abanico de tareas y responsabilidades del cuidado de los niños/as en la actualidad es amplísimo y sus contornos son difusos, pero tienen en común la exigencia de una cada vez mayor atención y esfuerzo (Gómez Espino, 2012). Estas responsabilidades, su ejercicio y resultados, tienen un peso importante en la construcción de la identidad individual, especialmente en el caso de las madres. La modernización reflexiva conlleva la constante toma de decisiones, con demasiada frecuencia sin tiempo ni información suficientes para valorarlas, donde el responsable único — de los errores y aciertos — es el mismo individuo (Beck y Beck-Gernsheim, 2003; Lash, 2003). Y eso genera una percepción muy aguda de la incertidumbre y con ésta del malestar. La maternidad no escapa al peso de esta responsabilidad, al contrario, parece que la exacerba pues las consecuencias de las decisiones no son asumidas únicamente por las madres (y padres) sino por sus hijos/as. No es extraño que se opte tantas veces por un cuerpo de expertos que aporten recetas que puedas contribuir a aliviar esa angustia.

Obviamente, estas prescripciones normativas han generado, y generan, interpretaciones, apropiaciones e incluso resistencias diversas (Bolufer, 1995). En este sentido la posición que se ocupe en el mercado de trabajo es básica en el acceso a recursos económicos, pero también temporales y culturales que se puedan dedicar a la crianza, sobre todo ante su profunda precarización que va excluyendo cada vez a más personas de unas mínimas condiciones de estabilidad y seguridad (Standing, 2013). Trabajo remunerado y crianza requieren hoy de las personas, de las mujeres sobre todo, de una atención constante e intensa y de inversiones de recursos equiparables (Hays, 1998; Blair-Loy, 2001; Christopher, 2012). Investigaciones realizadas en el marco

\footnotetext{
Según la Encuesta de Usos del Tiempo 2009-2010 (INE, www.ine.es) las mujeres dedican a diario dos horas más que los hombres al cuidado.

En 2020 las excedencias laborales por cuidado familiar en la Comunidad Valenciana fueron en un $93 \%$ solicitadas por mujeres en el caso de los hijos/as y un $81 \%$ de otros familiares (elaboración propia a partir de los datos del Anuario de Estadísticas Laborales, 2020. https://www.mites.gob.es/es/estadisticas/anuarios/2020/ index.htm. Consulta: 31.08.2021)..
} 
norteamericano muestran que, a pesar del incremento de las parejas de doble ingreso y de las demandas del mercado de trabajo, las madres han mantenido, en general, el volumen de tiempo que le dedican a los hijos/as y el de los padres se ha visto incrementado (Bianchi, 200, 2011). Y este tiempo, además está marcado por otras variables como el nivel educativo: a mayor nivel de estudios más tiempo con los hijos e hijas (Dotty y Treas, 2016). Resultados similares se han encontrado en otros países (Sayer et al., 2004) a costa de la minimización del tiempo propio de madres y padres. Y ante esta coincidencia de demandas sobre un único sujeto es fácil la colisión, sobre todo si la solución legítima pasa por los arreglos individuales, donde el azar de contar con los recursos necesarios (ya sea tener un jefe flexible o una red de apoyo suficiente) y la decisión personal ante el dilema de familia-trabajo remunerado prima ante consideraciones estructurales (Christopher, 2012; Edgley, 2021).

Los fundamentos en los que descansa la maternidad intensiva se han generalizado, convirtiéndose en el estándar de la concepción del cuidado de los niños y niñas. Pero este estándar requiere el acceso al capital —económico, cultural, social y emocional— propio de la clase media (Hays, 1998; Gillies, 2005, Elliot et al., 2015). Género, clase y raza se entrecruzan ${ }^{8}$ y ofrecen un mayor o menor abanico de recursos para poder llevar a la práctica los requerimientos de la maternidad/crianza intensiva, penalizando, material y simbólicamente a quienes no alcanzan los ideales de cuidado imperantes (Gillies, 2005; Elliot et al., 2015).

\section{Jornada escolar continua como recurso de la maternidad intensiva}

A pesar de la diversidad de argumentos a favor, en el núcleo central de la defensa de la jornada continua podemos encontrar la importancia de limitar la vinculación de los niños/as con la escuela en un tiempo muy acotado, evitando que el tiempo escolar y periescolar se alargue durante todo el día (Morán de Castro y Caride, 2005). Una disponibilidad temporal que vista desde la perspectiva que ofrece la ideología de la maternidad intensiva permitiría cumplir mejor con las que hoy se consideran necesidades y ritmos de los niños/as: mayor tiempo de convivencia familiar, un discurrir del tiempo más pausado, mayor capacidad para ofrecerles otras actividades y recursos educativos y de juego.

\section{1. ¿Menos escuela, para más familia?}

Uno de los principales argumentos por los que se ha defendido el cambio de jornada escolar, tanto por parte de las familias, pero también de sus docentes como voz de autoridad, ha sido la necesidad de pasar más tiempo con los hijos/as'. Concentrar las horas presenciales de escuela durante la mañana y liberar la tarde, incluso evitar la necesidad de utilizar el servicio de comedor, ha supuesto para muchas familias hacer posible el aumento del tiempo disponible para la convivencia familiar, así como la realización de otras actividades y se ha convertido en una justificación fundamental para defender este cambio de jornada.

De hecho, favorecer la comunicación entre padres e hijos así como la implicación activa de los padres y madres en la educación de los mismos es también una idea repetida a lo largo de los proyectos específicos de organización horaria consultados ${ }^{10}$. No en vano, la participación

8 También la monoparentalidad, paradójicamente con menores recursos por convertirse en madres (Obiol-Francés y Almeda, 2018).

9 En un artículo pro-jornada continua un maestro lo expresaba claramente: «en muchos casos lo que necesitan los alumnos son padres que pasen tiempo con ellos» (Ruiz, 2021: 38).

10 Resulta complicado el análisis de los Proyectos Específicos de Organización de la Jornada Escolar de los diferentes centros pues no existe ningún repositorio donde 
de las familias en las diferentes dimensiones de la vida escolar es un elemento tradicionalmente reclamado bajo la creencia que puede resultar beneficiosa para incrementar el rendimiento académico de los niños/as. Esta participación puede ser tanto colectiva como individual, aunque en todos los casos gira en torno a una relación no siempre plácida entre las familias y el cuerpo docente. De manera mayoritaria es una relación construida desde la escuela, por parte de los docentes, y a medida de sus requerimientos con el fin de desarrollar hábitos que consideran propicios para la misma (Cárcamo y Garreta, 2020). En esta subordinación de las familias a los parámetros marcados por los docentes, se ha de tener en cuenta la debilidad del tejido asociativo de las familias en la escuela, lo que ha sido fundamental, por otro lado, para la expansión de la jornada continua (Fernández Enguita, 2001).

Sin embargo, en las propuestas elaboradas en los centros valencianos para optar al cambio de jornada escolar no se demanda tanto la participación de las familias en la escuela, sino abrir la posibilidad de la implicación en la formación como individuo a través de la convivencia familiar.

\begin{abstract}
«El hecho de tener toda la tarde a su disposición puede permitir que niños y familias compartan más tiempo juntos, planeen mejor su estudio y ocio de tal forma que se reduzcan los niveles de estrés, aumente el nivel de felicidady, en consecuencia, su salud mental sea mejor». (Proyecto específico. CEIP El Romeral, Alcoi, pág. 17, traducción propia).
\end{abstract}

De hecho, según la normativa vigente, uno de los ámbitos de justificación de la necesidad de abordar en los centros de primaria el cambio de jornada escolar era la conciliación de la vida familiar y laboral ${ }^{11}$. En la normativa, como en el caso de otras comunidades autónomas, se exige a los centros ofrecer un programa de actividades extraescolares que garanticen que los niños/ as puedan permanecer en el centro por las tardes. En el caso valenciano se ha determinado un tiempo — hasta las $17 \mathrm{~h}$. — de actividades obligatorias para los centros, gratuitas y voluntarias para las familias. Esta inclusión conlleva que existan tres posibilidades de recoger a los niños/ as de la escuela: a las 14h, tras el periodo lectivo, a las 15:30h, tras la hora del comedor, o bien a las $17 \mathrm{~h}$ tras las actividades voluntarias. Además, claro, de la hora de recogida tras las actividades extraescolares de centro, organizadas por el AMPA. Esta flexibilidad horaria es uno de los principales elementos que los proyectos de los centros defienden como herramienta fundamental a favor de la conciliación familiar y laboral ${ }^{12}$.

No se trata de una demanda extraña. El ritmo acelerado de vida actual, especialmente con la proliferación de las parejas de doble ingreso, así como la precariedad laboral basada en intensas y elevadas demandas del mercado de trabajo tanto en tiempo con largas jornadas como en atención y disponibilidad, conlleva la percepción de que se necesita más tiempo para la familia. Los datos nos muestran que en el Estado español en 2021 un 50,7\% (un 61,7\% en el caso de los hombres, un $37,8 \%$ entre las mujeres $)^{13}$. Es decir, más de la mitad de las personas ocupadas rea-

sean accesibles, a veces ni tan siquiera están contenidos en la página web de los centros. Se trata de proyectos muy semejantes unos a otros, en los que se repiten ideas, propuestas e incluso párrafos. Hemos de entender la capacidad de homogenización que tienen los diferentes foros y redes que comparten los maestros/as así como el papel jugado por sus sindicatos, entre cuyos servicios a ofrecer en alguna ocasión encontramos apoyo para la elaboración de estos proyectos.

11 Siendo el resto de los ámbitos requeridos el pedagógico, el de coordinación, el de convivencia, el de coeducación e igualdad de género y el de salud.

12 CEIP Vicente Gaos, València.

13 Encuesta de Población Activa, 2021 ( $2^{\circ}$ trimestre). INE, www.ine.es (consulta 31.08.2021). 
lizaron un total de horas efectivas trabajadas durante una semana de 40 horas o más. El mercado de trabajo hace un uso muy intenso del tiempo de los trabajadores/as, con la asunción generalizada de la disponibilidad total al trabajo remunerado tanto en trabajos poco cualificados como en el caso de las profesiones liberales y académicas, como hemos señalado en estudios previos en la realidad valenciana (Obiol-Francés, 2010; Obiol-Francés et al, 2017; Villar y Obiol-Francés, 2018; Obiol-Francés et al. 2019).

En definitiva, la jornada continua deja entrever un interés en limitar el tiempo que el tiempo lectivo ocupa en la jornada diaria de los niños/as y justificar este acotamiento ante la necesidad que identifican en los más jóvenes de pasar más tiempo con su familia, sea cual sea su edad (en el periodo comprendido entre los 2 a los 12 años) y sean cuales sean sus circunstancias familiares, sociales y residenciales. Sin embargo, los análisis realizados apuntan a que se da si existen las condiciones — sobre todo de carácter socioeconómico - para que los progenitores dispongan de tiempo libre para compartirlo (Fernández Enguita, 2001; Sintes, 2012). Porque es importante entender que para pasar tiempo con la familia se ha de tener una familia, y esa familia ha de estar presente: «esta mejora de la vida familiar se da si, y sólo si, existen las condiciones previas, es decir, si hay una familia esperando» (Fernández Enguita, 2001: 99; cursiva del autor). De igual manera, Morán de Castro y Caride (2005) señalan que el tiempo dedicado a las relaciones familiares no se altera significativamente de los centros con jornada partida a los de jornada continua. Los mismos defensores de la jornada continua lo expresan crudamente: «De esta forma [con la jornada continua], muchas familias disfrutarán de sus hijos por las tardes, si es que pueden, quieren y tienen la suerte de trabajar con jornada intensiva de mañana» (Ruiz, 2021:38). Sin embargo, no se trata de suerte sino de estructura social.

\subsection{Menos tiempo de escuela, más tiempo disponible para otras actividades formativas}

En relación a la liberación del tiempo de las tardes de la jornada escolar lectiva se encuentra la posibilidad proclamada de realizar, con más calma y de manera más óptima, actividades extraescolares, tanto en el centro mismo como en otros lugares del municipio. De hecho, en el caso de los proyectos presentados por los centros escolares valencianos son varios los que integran a otros actores (ayuntamientos, asociaciones, clubes deportivos) en la programación de sus actividades extraescolares, tanto las que son gratuitas como las que se generen tras las $17 \mathrm{~h}$. de la $\operatorname{tarde}^{14}$.

Se vislumbra que los centros y la administración entienden que las actividades son un elemento de interés para las familias. Se le atribuyen diversos beneficios a la realización de actividades extraescolares como la mejora en la salud y condición física de los niños/as, la mejora en sus relaciones sociales y conducta así como efectos positivos en el rendimiento académico (Fraguelas et al., 2021: 96). Fernández Enguita (2001: 89-90) también se refiere, al estudiar la expansión de la jornada continua, a los principales beneficios que se le atribuyen a las actividades extraescolares: permiten adquirir formación en aquellas áreas que no acaban de ser bien cubiertas por la escuela — como las disciplinas artísticas o las actividades deportivas-, contribuyen a fortalecer

14 Proliferan la proyección de películas o vídeos didácticos, el apoyo en el estudio, los talleres de lectura o plástica... Es importante señalar se incluye, en muchos casos, la advertencia de la necesidad de que el alumnado asista regularmente (CEIP Azorín de Catral y CEIP Cap d'Or de Moraira). 
el aprendizaje de conocimientos que se intuyen importantes para el mercado de trabajo, como es el caso de las lenguas extranjeras, pueden fomentar el interés por el estudio además que permiten la prueba y el error en la apertura de nuevos itinerarios formativos y, por último, pueden funcionar como una herramienta para hacer compatible trabajo y familia, aunque en este último aspecto el estudio de Cardús et al., (2003) introduce matices, dado que pueden ser un elemento de distorsión con horarios totalmente incompatibles.

Por otro lado, si atendemos a los datos internacionales sobre asistencia a actividades extraescolares, como ya presentamos en estudios anteriores (Gabaldón-Estevan y Obiol-Francés, 2017: 47), el alumnado español asiste en gran medida. Según datos de PISA (2016, referidos a alumnado de 15 años de edad) de los 28 países que entran en la comparativa, España ocupa el tercer lugar en grado de asistencia a tiempo de estudio con un tutor personal, es el quinto país en presencia en academias de repaso y la séptima en deberes escolares. Aunque esta situación contrasta con la escasa asistencia a actividades o servicios donde el cuidado es un elemento central. España es el país que, según los datos de la $\mathrm{OCDE}^{15}$, menor tasa de asistencia presenta: los niños y niñas de entre 6 y 11 años participan una media de un $5 \%$ mientras que la media de la OCDE es de un 28,6\% y la de la Unión Europea de un 28,3\%. En tiempo esta participación se traduce en 5,9 horas a la semana, mientras que la media de los países de la OCDE está en 9,2 horas/semana. Unos datos que se encuentran influidos por el nivel de estudios de la madre: la participación en actividades extraescolares de cuidado es de un 6,1\% en el caso de madres con estudios superiores frente a un 3,7\% de hijos de madres sin éstos. También en el caso del nivel de renta disponible familiar, siendo las familias que se sitúan en el $3^{\text {er }}$ tercil —en el tramo más elevado- las que participan en un 6,2\%, mientras que las que se sitúan en el $1^{\text {er }}$ tercil —en el tramo más bajo- lo hacen en un 3,4\%. Aunque se necesitarían más datos para conocer con mayor precisión el grado de asistencia y cómo ésta se encuentra condicionada a la naturaleza de las actividades, entre otros factores.

La capacidad para guiar a los niños/as en este tiempo no está igualmente distribuida. Como señala Giménez Nadal (2013), a mayor nivel educativo de las madres, un mayor tiempo dedicado a lo que denominan cuidado infantil de contenido educativo. Igualmente, las niñas y niños de familias con situaciones socioeconómicas más desfavorecidas acaban realizando actividades con menor valor educativo y más sedentarias (Sintes, 2012). O, como han evidenciado Bonal y González (2021) en su análisis sobre la educación durante el confinamiento, el capital cultural y económico influyen significativamente en la capacidad de desarrollar actividades de aprendizaje que la escuela considere válidas. De hecho, aunque el interés y preocupación por la relación de los hijos/as con la escuela es general, en el caso de las familias pocas veces coincide con los parámetros sancionados por la escuela como correctos (Gillies, 2006).

En la cuestión que aquí abordo, y a pesar de que todas las propuestas de cambio en la jornada escolar han venido acompañadas de una propuesta de expansión en número y social de las actividades extraescolares, el resultado con el paso del tiempo ha sido el opuesto: una reducción de las posibilidades (Fernández Enguita, 2001). Además, también se ha acentuado el desplazamiento de la escuela ante el mercado en la organización de estas actividades y eso hace todavía

15 Datos referidos al año 2017. https://www.oecd.org/els/family/PF4-3-Out-of-school-hours-care.pdf Consulta 09.09.2021. 
más complicada la participación de las clases más desfavorecidas (Morán de Castro, 2012), sobre todo de aquellas actividades que pueden tener más interés y ser de mayor calidad. De hecho, el estudio realizado por Fernández Enguita (2001) da como resultado que las familias más acomodadas con la jornada continua huyen del centro para realizar estas actividades en otros lugares.

En conclusión, aunque la participación de las actividades extraescolares es uno de los argumentos fundamentales para la defensa del cambio en la jornada escolar, los resultados parecen no acompañar. Al menos no de manera generalizada, sí en el caso de las familias más acomodadas que tienen más tiempo disponible para poder acudir a aquellas actividades que consideren de interés para maximizar la formación de sus hijos. Tal y como coinciden Feito (2007) y Fernández Enguita (2001) la defensa de la jornada continua representa para algunas familias más acomodadas una vía para maximizar los resultados de su inversión educativa al limitar la mañana para las actividades lectivas y dejar las tardes completas para poder optar a otras actividades formativas que no se ofrecen en los centros y que son valoradas socialmente. Podríamos considerar a la jornada continua como un camino más sencillo hacia la distinción social: «La clase media ha encontrado en la jornada continua una forma mejor de combinar el mínimo común que provee la escuela pública con el plus que se ofrece en el mercado». (Fernández Enguita, 2001: 94).

\section{Maternidad intensiva y jornada continua: reflexiones finales}

En este artículo he tratado de aproximarme al proceso de cambio de jornada escolar en el que he estado interesada desde sus inicios en la Comunidad Valenciana (Gabaldón-Estevan y Obiol-Francés, 2017) a partir de la relación de este cambio con las normas culturales de la sociedad occidental acerca de la maternidad y del cuidado de los niños y niñas. Consciente que el proceso responde a razones diversas y complejas me interesa conocer los puntos de convergencia de la llamada «ideología de la maternidad intensiva» (Hays, 1998) con los argumentos de defensa de la conveniencia de la jornada escolar continua. $\mathrm{Y}$ aunque el análisis realizado abre más preguntas que respuestas, considero que logra apuntar algunas cuestiones de interés.

En primer lugar, en el proceso de expansión de la jornada continua la escuela ha jugado un papel fundamental —utilizando su autoridad ante las familias — en la diseminación de la ideología de la maternidad intensiva y en la promoción de su transversalidad entre las familias, sea cual sea su extracción social. En su defensa de la jornada continua han utilizado el argumento por el cual los niños/as son seres puros, con ritmos y necesidades muy concretas que se han de atender y a los que los progenitores, específicamente sus madres, han de proteger ante una sociedad — la escuela, en este caso- que puede perjudicarles. Lo que no deja de ser paradójico pues es la misma escuela la que reclama un papel menor en la sociedad y en la cotidianidad de los niños y niñas.

El resultado ha sido que las familias con menos recursos estarían aceptando —e incluso promoviendo- un cambio en la organización horaria, pues si no fuera así difícilmente la jornada continua se hubiera extendido como lo ha hecho entre los centros escolares de la red pública de la Comunidad Valenciana. Y eso a pesar de que, según las evidencias acumuladas, les perjudica: a más tiempo de escuela, menos desigualdad, puesto que el tiempo fuera de la escuela — ya se traduzca en tiempo familiar o tiempo de formación y cuidado- está claramente mediatizado por variables como la clase o el género. En este sen- 
tido, la maternidad intensiva, y por extensión la crianza intensiva, está jugando un papel importante en la distinción de las familias más acomodadas. Sería importante conocer en qué grado, pero también cómo las familias con menos recursos se apropian del concepto de «maternidad intensiva» y cómo les afecta.

En segundo lugar, la jornada continua viene a reforzar la idea de la responsabilidad individual, sobre todo de las mujeres, ante el cuidado de los hijos/as. La sensación de responsabilidad que se ha agudizado en los últimos años ante la inseguridad ontológica que vivimos y el cambio de nuestras fuentes de sentido y de construcción de la identidad individual impregna también la crianza y la educación de los hijos/as. Ha crecido la percepción de la responsabilidad individual de las madres, y por extensión de los padres, en la protección del bienestar físico y emocional de las hijas/os en el presente, pero también en el futuro. Una responsabilidad que demanda esfuerzos económicos, de tiempo y emocionales, pero siempre desde la perspectiva de la elección, puesto que la maternidad es hoy una opción.

La jornada continua amplía el abanico de las decisiones. Ahora se ha de decidir acerca de cuándo recoger a los hijos/as de la escuela, si es o no conveniente que hagan uso del servicio de comedor y también de las actividades que se proporcionan en el marco del centro educativo. Igualmente se ha de decidir cómo llenar (si es que se decide llenar) el tiempo de la tarde. Y con cada elección se incrementa el peso de la responsabilidad de acertar (o no) y por tanto de ser considerada «una buena madre».

Y en último lugar, y en estrecha relación con el punto anterior, la jornada continua viene a reforzar la ideología de la maternidad intensiva y eso ha de generar distorsiones importantes entre las demandas del mercado de trabajo y las de la crianza, ambas cada vez más grandes y rígidas, entre las madres. Es cierto que, en el caso valenciano, los niños/as pueden quedarse hasta las 17h. en la escuela, pero el progresivo vaciamiento del servicio de comedor y de las actividades gratuitas que parece acompañar a la jornada continua según los estudios realizados, así como una más que probable disminución de su calidad, ha de conllevar que los niños/as sean recogidos a mediodía. Al menos si se atiende a los valores imperantes bajo la lógica de la maternidad intensiva, penalizando además, en este mismo sentido, a quienes no lo hagan.

Esta situación incrementaría las dificultades ya enormes de conciliar el trabajo remunerado con el cuidado y atención de los hijos/as, una circunstancia que todavía recae mayoritariamente en las mujeres, haciendo más vulnerable su posición laboral. Sería muy interesante poder analizar esta situación desde lo que McRobbie (2007, 2010) y Gill (2007) en sus análisis de la cultura occidental, han denominado «sensibilidad postfeminista»: entender que estas dificultades de conciliar familia y trabajo remunerado ya no se deben a una cuestión de desigualdad estructural sino de elección personal y por tanto se han de resolver en el ámbito del individuo, lo que beneficia y hace perdurar el actual sistema capitalista.

En conclusión, el análisis del proceso de cambio en la jornada escolar desde la perspectiva familiar ofrece elementos de gran interés ante una realidad que se va extendiendo y consolidando a pesar de que los estudios existentes muestran su escasa conveniencia.

\section{Referencias bibliográficas}

Aguado, Ana María (1998): “Treball, gènere i identitat femenina a la societat valenciana contemporània”. Cuadernos de Geografía, 64, 325-337.

Badinter, Elisabeth (2011). La mujer y la madre: un libro polémico sobre la maternidad como nueva forma de esclavitud. Madrid: La esfera de los libros. 
Barbeta-Viñas, Marc y Cano, Tomás (2017): “¿Hacia un nuevo modelo de paternidad? Discursos sobre el proceso de implicación paterna en la España urbana”. Revista Española de Investigaciones Sociológicas, 159: 13-30. DOI: http://dx.doi.org/10.5477/cis/reis.159.13.

Beck, Ulrich y Beck-Gernsheim, Elisabeth (2003). La individualización. El individualismo institucionalizado y sus consecuencias sociales y políticas. Barcelona, Paidós.

Beck-Gernsheim, Elisabeth (2003). La reinvención de la familia. En busca de nuevas formas de convivencia. Barcelona, Paidós.

Bianchi, Suzanne (2000): "Maternal employment and time with children: Dramatic change or surpring continuity?”. Demography, 37, 401-414. DOI: http://dx.doi.org/10.1353/dem.2000.0001.

Bianchi, Suzanne (2011): "Family Change and Time Allocation in American Families". The Annals of the American Academy of Political and Social Science, 638, 21-44. DOI: https://doi.org/10.1177/0002716211413731.

Blair-Loy, Mary (2001): “Cultural Constructions of Family Schemas: The Case of Women Finance Executives”. Gender and Society, 15, 5, 687-709. DOI: https://doi.org/10.1177/089124301015005004.

Bolufer, Mònica (1995): “La construcción de la identidad femenina: Reformismo e Ilustración”. Estudis: Revista de bistoria moderna, 249-265.

Bolufer, Mònica (2010): "Madres, maternidad: Nuevas miradas desde la historiografía" en Gloria A. Franco Rubio (ed.): Debates sobre la maternidad desde una perspectiva histórica (siglos XVI-XX). Madrid: Icaria Editorial.

Bolufer, Mònica (2013): “Ciència i moral: en els orígens de la maternitat totalitzant”. Mètode: Revista de difusió de la investigació,76, 70-75. DOI: https://doi.org/10.7203/metode.76.2067.

Bonal, Xavier y González, Sheila (2021). "Educación formal e informal en confinamiento: una creciente desigualdad de oportunidades de aprendizaje". Revista de Sociología de la Educación (RASE), 14 (1), 44-62. DOI: https://doi.org/10.7203/RASE.14.1.18177.

Cárcamo, Héctor y Garreta, Jordi. (2020): "Representaciones sociales de la relación familia-escuela desde la formación inicial del profesorado". Revista Electrónica de Investigación Educativa, 22, e11, 1-14. DOI: https://doi.org/10.24320/redie.2020.22.e11.2406.

Cardús, Salvador; Pérez, Carmen y Morral, Sergi (2003). Propostes d'intervenció per a la conciliació d'horaris familiars, escolars i laborals. Barcelona: Generalitat de Catalunya.

Christopher, Karen (2012): “Extensive mothering: Employed mothers' constructions of the good mother”. Gender and Society, 26(1), 73-96. DOI: https://doi.org/10.1177/0891243211427700.

Del Olmo, Carolina (2013). ¿Dónde está mi tribu?: maternidad y crianza en una sociedad individualista. Madrid: Clave Intelectual.

Dotti Sani, Giulia M. y Treas, Judith (2016): “Educational Gradients in Parents' Child-Care Time Across Countries, 1965-2012”. Fam Relat, 78: 1083-1096. DOI: https://doi.org/10.1111/jomf.12305. 
Edgley, Alison (2021): "Maternal Presenteeism: Theorizing the Importance for Working Mothers of 'Being there' for their Children Beyond Infancy”. Gender, Work and Organization, 28 (3), 1023-1039. DOI: https://doi.org/10.1111/gwao.12619.

Elliot, Sinikka; Powell, Rachel y Brenton, Joslyn (2015): “Being a Good Mom: Low-Income, Black Single Mothers Negotiate Intensive Mothering”. Journal of Family Issues, 36 (3), 351-370. DOI: https://doi. org/10.1177/0192513X13490279.

Feito, Rafael (2007): “Tiempos Escolares. El debate sobre la jornada escolar continua y partida”. Cuadernos de Pedagogía, 365, 74-79.

Fernández Enguita, Mariano (2001). La jornada escolar. Barcelona: Ariel.

Fraguela Vale, Raúl; Morán de Castro, Carmen y Varela Garrote, Lara (2020): "Entre lo escolar y lo extraescolar: nuevas realidades, viejos desafíos”. en José Antonio Caride Gómez, María Belén Caballo Villar, Rita Gradaçilles Pernas. (coord.): Tiempos, educación y ocio en una sociedad de redes. Barcelona: Octaedro.

Gabaldón-Estevan, Daniel y Obiol-Francés, Sandra (2017): “Guía sobre tiempos escolares”. Creativity and Educational Review (CEIR), 1, 12-69. DOI: https://doi.org/10.7203/CREATIVITY.1.12062.

Gálvez, Lina (2013): “Una lectura feminista del austericidio”. Revista de economía crítica, 15, 80-110.

Gálvez, Lina y Rodríguez-Modroño, Paula (2016): "Una crítica desde la economía feminista a la salida austericida de la crisis". Atlánticas. Revista Internacional de Estudios Feministas, 1 (1), 8-33. DOI: http:// dx.doi.org/10.17979/arief.2016.1.1.1346.

Gill, Rosalind (2007): "Postfeminist Media Culture: Elements of a Sensibility". European Journal of Cultural Studies, 10 (2), 147-166. DOI: https://doi.org/10.1177/1367549407075898.

Gillies, Val (2005): "Raising the 'Meritocracy': Parenting and the Individualization of Social Class". Sociology, 39 (5), 835-853. DOI: https://doi.org/10.1177/0038038505058368.

Gillies, V (2006): “Working Class Mothers and School Life: Exploring the Role of Emotional Capital”. Gender and Education, 18:3, 281-293. DOI: https://doi.org/10.1080/09540250600667876.

Gómez Espino, Juan Miguel (2013): “Two Sides of Intensive Parenting: Present and Future Dimensions in Contemporary Relations Between Parents and Children in Spain”. Childhood, 20 (1), 22-36. DOI: https://doi.org/10.1177/0907568212445225.

Hays, Sharon (1998). Las contradicciones culturales de la maternidad. Barcelona: Paidós.

Lash, Scott (2003): "Individualización a la manera no lineal. Prefacio" en Ulrich Beck, Elisabeth Beck Gernsheim (eds.): La individualización. El individualismo institucionalizado y sus consecuencias sociales y politicas. Barcelona: Paidós.

McRobbie, Angela (2007): “Top Girls?”. Cultural Studies, 21 (4-5), 718-737, DOI: https://doi. org/10.1080/09502380701279044. 
Medina Bravo, Pilar; Figueras-Maz, Mònica y Gómez-Puertas, Lorena (2014): "El ideal de madre en el siglo xxi. La representación de la maternidad en las revistas de familia". Estudios Sobre El Mensaje Periodístico, 20 (1), 487-504. DOI: https://doi.org/10.5209/rev_ESMP.2014.v20.n1.45244.

Morán de Castro, Carmen (2012): “Incidencia de la jornada escolar en los procesos de socialización infantil”. Educar em Revista, 45, 19-36.

Morán de Castro, Carmen y Caride, José Antonio (2005): "La jornada escolar en la vida cotidiana de la infancia”. Cuadernos de pedagogía, 349, 64-69.

Morán de Castro, Carmen y Cruz López, Laura (2011): "Vida cotidiana, tiempos escolares y derechos de la infancia”. Educación Social, 47, 84-94.

Naldini, Manuela (2003). The Family in the Mediterranean Welfare States. Londres: Frank Cass.

Obiol-Francés, Sandra (2010). Teixir certeses. Percepcions i respostes a la incertesa dels treballadors del textil-confecció a l'Alcoià, el Comtat i la Vall d'Albaida. Barcelona: Universitat Autònoma de Barcelona. Tesis doctoral. (www.tdx.cat).

Obiol-Francés, Sandra y Santos-Ortega, Juan Antonio; Villar-Aguilés, Alìcia; Muñoz-Rodríguez, David y Querol, Vicent (2017): “Crisis de las clases medias y cambios en los profesionales liberales independientes. Abogados, arquitectos y periodistas”. Sociología del Trabajo, 89, 75-92.

Obiol-Francés, Sandra; Almeda, E. (2018): “Las contradicciones del cambio familiar". Encuentro científico intermedio e Internacional del Comité de Sociología del Género (FES). Perspectivas de género en la teoría, metodología, educación, salud, empleo y violencia. València: Comité de Sociología del Género. Federación Española de Sociología. Comunicación.

Obiol-Francés, Sandra; Santos-Ortega, Juan Antonio; Muñoz-Rodríguez, David (2019): “Job Precariousness Among Lawyers in Spain”. Journal of Poverty and Social Justice, 27 (2), 279-294. DOI: https:/ / doi. org/10.1332/175982718X15451304489459.

Rich, Adrienne (1996). Nacemos de mujer: la maternidad como experiencia e institución. Madrid: Cátedra.

Romero-Balsas, Pedro; Meil, Gerardo; Rogero-García, Jesús (2021): "Policemen on Leave Alone in Spain. A Rift in Hegemonic Masculinity?". Men and Masculinities, 24 (3), 483-500. DOI: https://doi. org/10.1177/1097184X19878221.

Ruiz Hidalgo, David (2021): “¿Jornada escolar? Continua, por supuesto”. Cuadernos de Pedagogía, 516, 3639.

Salido Cortés, Olga (2021): "Los efectos de la pandemia sobre la igualdad de género: algunos análisis sobre el mercado de trabajo español”. Panorama social, 33, 75-93.

Sayer, Liana C.; Bianchi, Suzanne M.; Robinson, John P. (2004): “Are Parents Investing Less in Children? Trends in Mothers' and Fathers' Time with Children”. American Journal of Sociology, 110 (1), 1-43. DOI: https://doi.org/10.1086/386270. 
Shirani, Fiona; Henwood, Karen y Coltart, Carrie (2012): "Meeting the Challenges of Intensive Parenting Culture: Gender, Risk Management and the Moral Parent". Sociology, 46 (1):25-40. DOI: https:/ / doi. org/10.1177/0038038511416169.

Sintes, Elena (2012). A les tres a casa? L'impacte social i educatiu de la jornada escolar contínua. Barcelona: Fundación Jaume Bofill.

Standing, Guy (2013). El precariado. Una nueva clase social. Barcelona: Pasado y Presente.

Villar-Aguilés, Alìcia y Obiol-Francés, Sandra (2018). Informe final de l'estudi sobre conciliació personal, familiar i professional de les persones que treballen a la Universitat de València.València: Unitat d'Igualtat. Universitat de València. Informe accesible en: https://www.uv.es/igualtat/webnova2014/ESTUDI_CONCILIACIO.pdf.

Visa Barbosa, Mariona; Crespo Cabillo, Cira (2012): "Las madres toman la palabra. Análisis del fenómeno de los blogs de madres” en Juan Carlos Suárez Villegas, Irene Liberia Vayá, Belén Zurbano-Berenguer (coord.): I Congreso Internacional de Comunicación y Género. Libro de Actas. Sevilla: Facultad de Comunicación. Universidad de Sevilla.

Visa Barbosa, Mariona (2019): "Relatos sobre maternidad, reproducción y crianza en la era post-televisión”. Investigaciones feministas, 10 (2), 281-294. DOI: https://doi.org/10.5209/infe.66494.

\section{Nota biográfica}

Sandra Obiol-Francés es profesora en el Departamento de Sociología y Antropología Social de la Universitat de València. Doctora por la Universitat Autònoma de Barcelona, miembro del grupo de investigación IPRODES (Universitat de València) y COPOLIS (Universitat de Barcelona) además del grupo de innovación docente CEFOCID-COPOLIS. Sus principales líneas de investigación son el análisis del cambio familiar desde una perspectiva de género, los procesos de precarización laboral y, más recientemente, la relación entre trabajo de cuidados y trabajo remunerado, en especial, en profesiones cualificadas. En relación a estos intereses ha participado en numerosos proyectos de investigación de los que se han derivado diversas publicaciones en revistas académicas, pero también ha participado activamente en actividades y publicaciones de divulgación científica. 